Case Report

\title{
A Giant Left Atrial Myxoma Neovascularized from the Right Coronary Artery
}

\author{
Demet Menekse Gerede, ${ }^{1}$ Irem Muge Akbulut, ${ }^{1}$ Sadik Ersoz, ${ }^{2}$ and Mustafa Kilıckap ${ }^{1}$ \\ ${ }^{1}$ Department of Cardiology, Cebeci Heart Center, Ankara University School of Medicine, Cebeci, 06590 Ankara, Turkey \\ ${ }^{2}$ Department of Cardiovascular Surgery, Ankara University School of Medicine, Ankara, Turkey \\ Correspondence should be addressed to Demet Menekse Gerede; drmeneksegerede@yahoo.com
}

Received 27 January 2015; Accepted 6 April 2015

Academic Editor: Aiden Abidov

Copyright (c) 2015 Demet Menekse Gerede et al. This is an open access article distributed under the Creative Commons Attribution License, which permits unrestricted use, distribution, and reproduction in any medium, provided the original work is properly cited.

\begin{abstract}
Myxomas are benign and the most common tumors of the cardiac muscle (Reynen, 1995). They are predominantly located in the left atrium. Clinical manifestations may vary according to the localization and the size of the myxoma. On the other hand, imaging of a myxoma by contrast dye during coronary angiography is a rare sign, which displays the vascular supply of the tumor. Here, we report the case of a 51-year-old man presenting with presyncope and palpitations due to a giant left atrial myxoma having its vascular supply from the right coronary artery (RCA).
\end{abstract}

\section{Case Presentation}

A 51-year-old man was referred to cardiology clinic due to presyncope attacks and palpitations. His medical history included type 2 diabetes mellitus, hypogonadism of unknown etiology, and hypertension. His physical examination was unremarkable. A transthoracic echocardiography was performed and revealed a giant left atrial mass attached to the interatrial septum with dimensions of $4.7 \times 6.7 \mathrm{~cm}$ (Figure 1 and Video 1 in Supplementary Material available online at http://dx.doi.org/10.1155/2015/614830), resulting in an average mitral transvalvular gradient of $8 \mathrm{mmHg}$. Echocardiographic findings indicated a suspicious diagnosis of left atrial myxoma. A preoperative coronary angiography was performed and revealed normal coronary arteries and the neovascularization of the mass from the conus branch of the right coronary artery (Figures 2 and 3). No other imaging study was performed prior to the surgery. The patient underwent surgery, which was carried out under cardiopulmonary bypass. A portion of the mass was excised by left atriotomy. The rest of the mass was removed by an incision to the interatrial septum. No attempts to mitral valve were performed during surgery.

The histopathologic examination of the mass was compatible with myxoma. A transesophageal echocardiography was performed after the surgery and showed an intact interatrial septum and mild mitral regurgitation. One week after the surgery, the patient was in good health and discharged from the hospital.

\section{Discussion}

Myxomas are the most frequent benign tumors of the heart. Approximately $85 \%$ of the myxomas are located in the left atrium [1]. The clinical manifestations usually depend on the anatomic position and size of the mass. There are mainly 3 types of presentations: embolic, obstructive, and constitutional. Embolic manifestations include visceral infarctions, stroke, and myocardial infarction. Obstructive manifestations are usually mistaken for valvular stenosis. For instance, mitral valve obstruction due to left atrial myxoma presents with symptoms such as syncope and dyspnea, mimicking mitral valve stenosis. It was previously shown that mitral stenotic effects occurred when the diameter of the mass exceeded 5 centimeters [2]. In our case the diameter of the tumor was $6.7 \mathrm{~cm}$, causing moderate mitral transvalvular gradient and presyncope attacks. Finally, myxomas may be associated with constitutional symptoms such as weight loss, malaise, fever, and loss of appetite. 


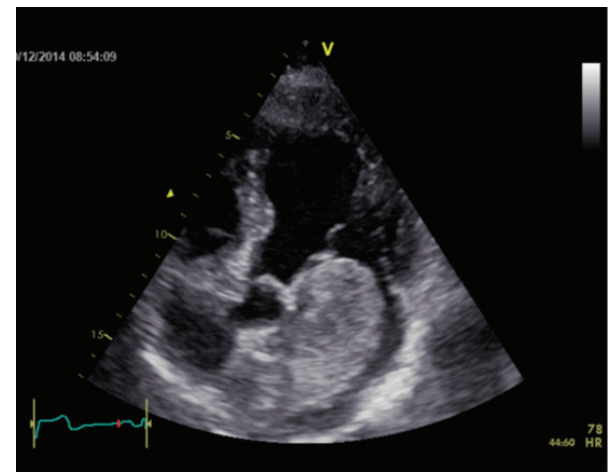

FIGURE 1: Transthoracic echocardiography shows the giant left atrial myxoma protruding through the mitral valve into the left ventricle during diastole.

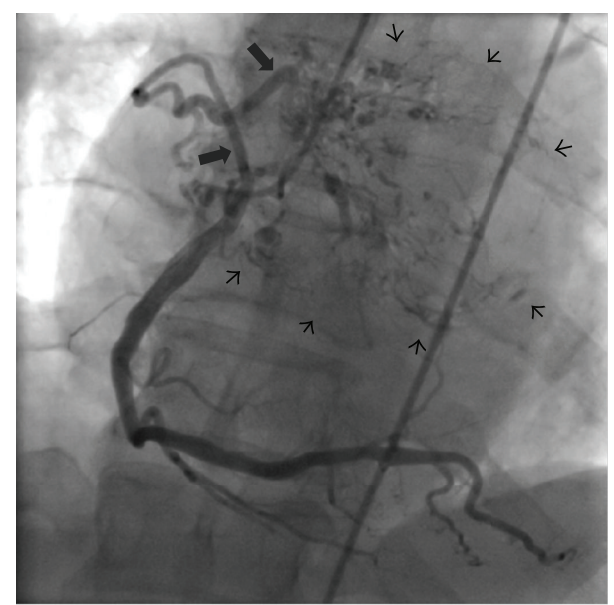

FIGURE 2: Coronary angiography image with a conus branch of the right coronary artery (large black arrows) vascularizing the myxoma with a wide area vascular network (small black arrows).

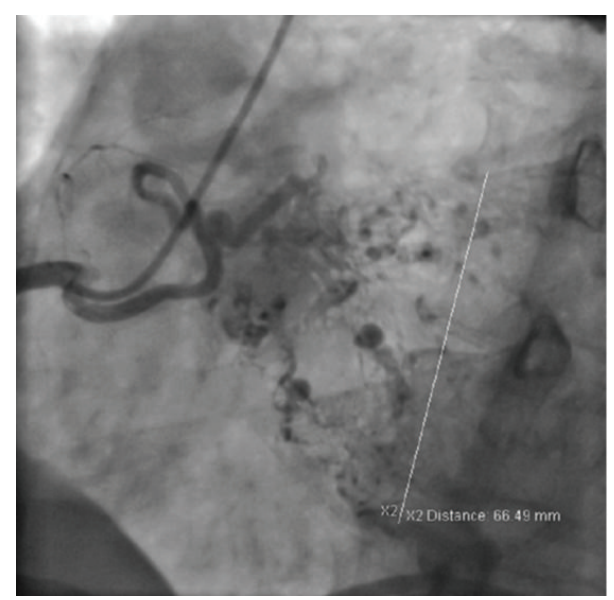

FIGURE 3: Right coronary angiogram with zoom mode shows diameter of left atrial myxoma vascularity by conus branch.
Beyond its value in ruling out coronary artery disease before surgery, a coronary angiography is also crucial for detecting the neovessels of the tumour. In the majority of cases, the supply of the vascularization is the circumflex artery, followed by the right coronary artery [3]. Similarly in our case, the right coronary artery was the source of the neovascularization.

Only a few patients with giant left atrial myxoma supplied from RCA were demonstrated [4-6]. Our patient, presenting with obscure symptoms, is a rare case of a giant left atrial myxoma, which was shown with contrast dye on RCA angiogram.

\section{Conclusion}

In conclusion, myxomas are rare, benign cardiac tumours. They can be presented with symptoms of obstruction when they are large in size, as in our case. Neovascularization can be seen in myxomas so that preoperative coronary angiograms should be performed in order to check for the coronary supply of the mass.

\section{Conflict of Interests}

The authors have no conflict of interests.

\section{References}

[1] K. Reynen, "Medical progress: cardiac myxomas," The New England Journal of Medicine, vol. 333, no. 24, pp. 1610-1617, 1995.

[2] A. Pucci, P. Gagliardotto, C. Zanini, S. Pansini, M. Di Summa, and F. Mollo, "Histopathologic and clinical characterization of cardiac myxoma: review of 53 cases from a single institution," American Heart Journal, vol. 140, no. 1, pp. 134-138, 2000.

[3] H. Omar, "The value of coronary angiography in the work-up of atrial myxomas," Herz, 2013.

[4] F. Burzotta, M. Pieroni, and E. Rossi, "Left atrial myxoma supplied from the right coronary artery," Heart, vol. 86, no. 2, article 206, 2001.

[5] M. Yazici, T. Norgaz, R. Akdemir, and S. Albayrak, "Asymptomatic giant left atrial myxoma supplied from right coronary artery in a 65-year-old woman," International Journal of Cardiology, vol. 101, no. 3, pp. 495-496, 2005.

[6] X. Wang, W. Ren, and J. Yang, "Neovascularized myxomacausing abnormal blood flow in the left atrium diagnosed by transesophageal echocardiography," Echocardiography, vol. 30, no. 1, pp. E10-E12, 2013. 


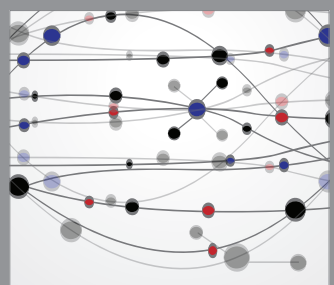

The Scientific World Journal
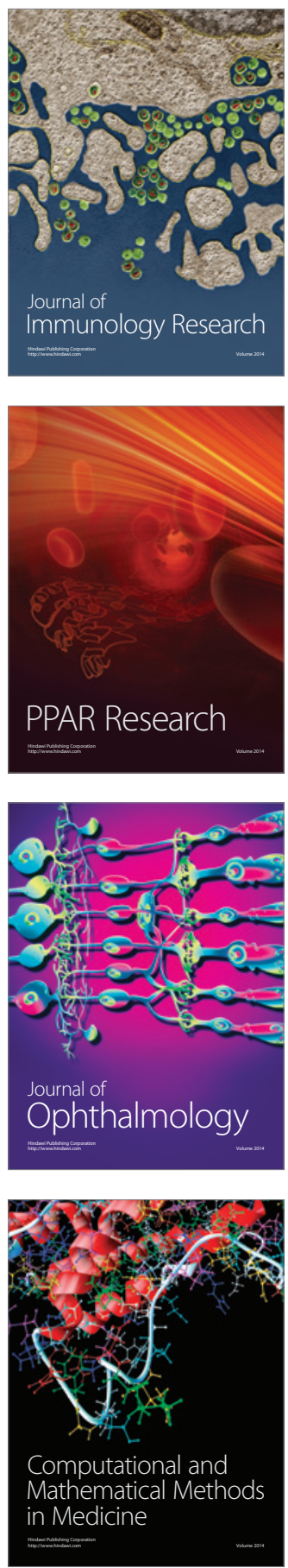

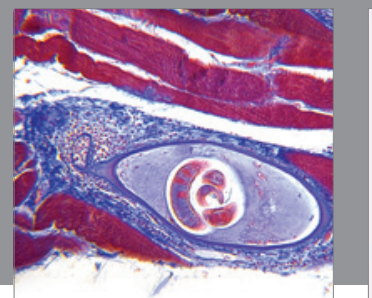

Gastroenterology

Research and Practice
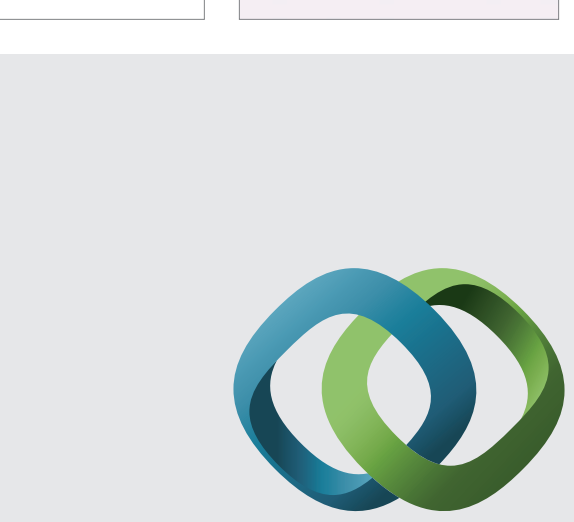

\section{Hindawi}

Submit your manuscripts at

http://www.hindawi.com
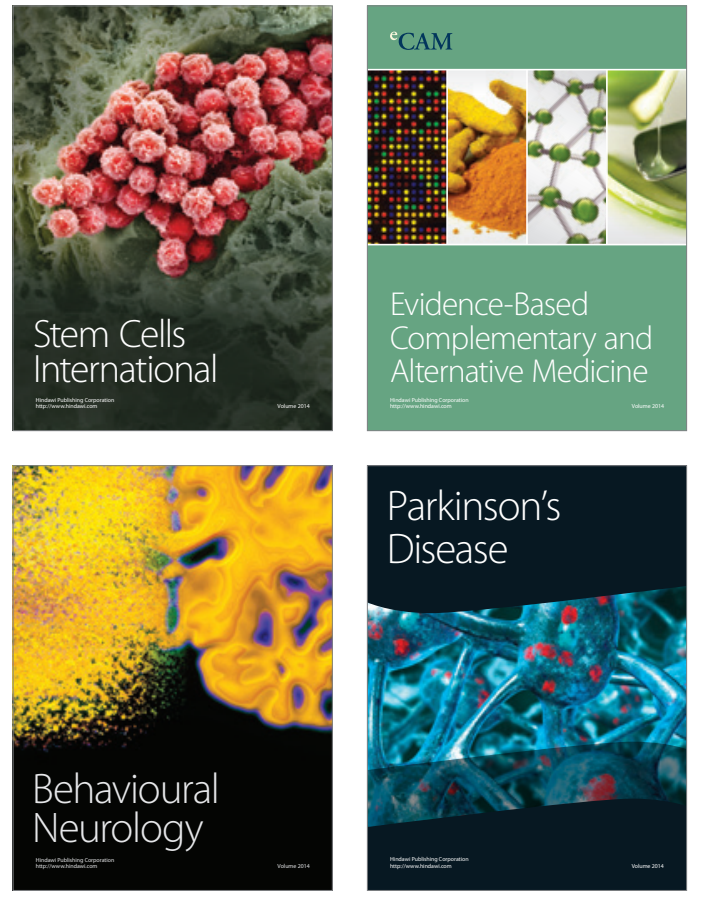
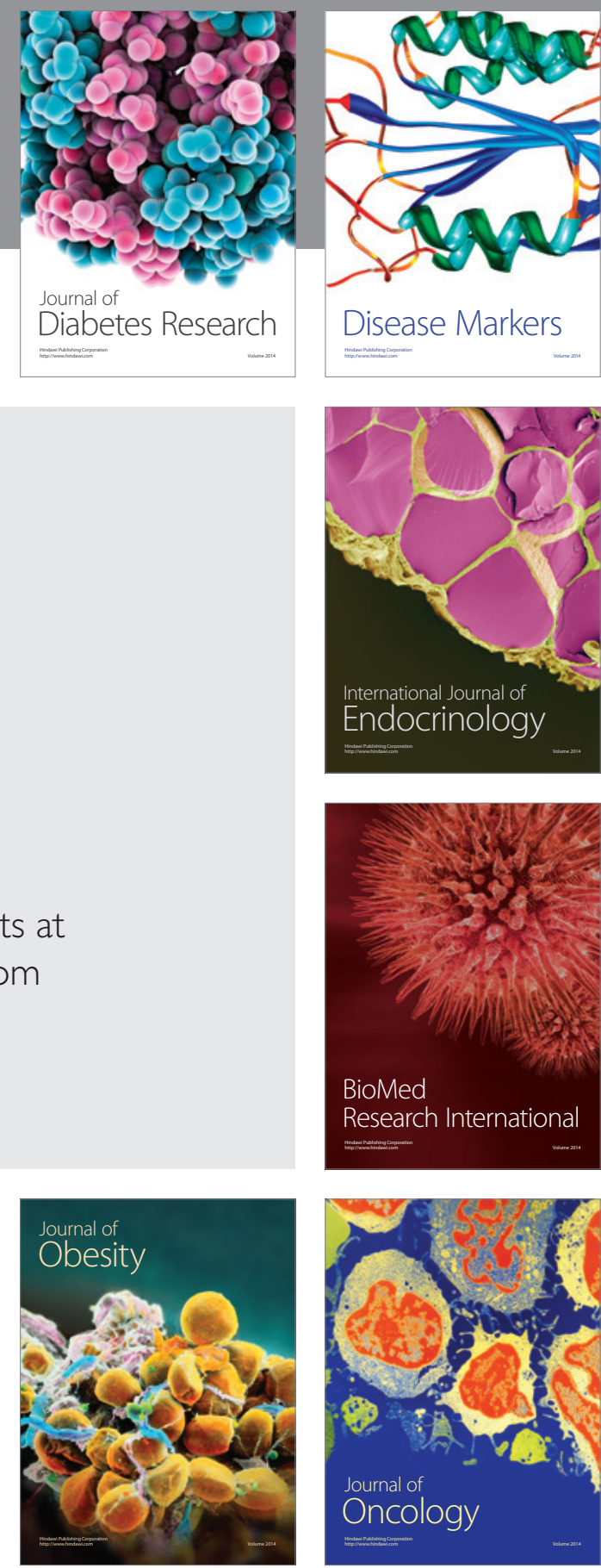

Disease Markers
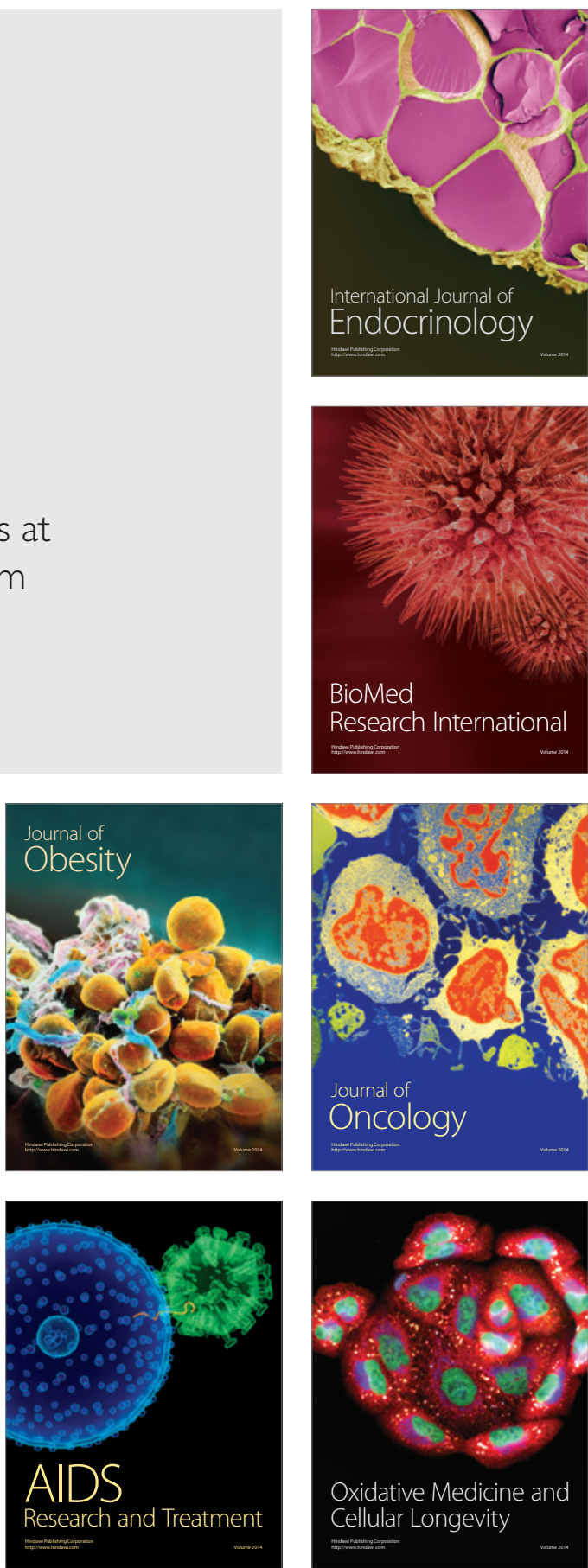